\title{
Research On Evaluation Index System of China's Inter-Provincial Electricity Market Operation
}

\author{
Zheng Zhao ${ }^{1, a}$, Changhai Yang ${ }^{2}$, Xiaoxuan Zhang ${ }^{1}$, Zhengying Liu ${ }^{2}$ and Yongcheng Liu ${ }^{2}$ \\ ${ }^{1}$ State Grid Energy Research Institute, Beijing, China \\ ${ }^{2}$ State Grid GanSu Electric Power Company Economic Research Institute, GanSu, China
}

\begin{abstract}
As China's electricity market develops rapidly in recent years, market evaluation turns out to be more necessary than before to make a comprehensive evaluation of market construction and operation, and ensure a fair and health electricity market. In this study, the international experience of market monitoring and assessment is firstly summarized. Then considering the feature of China's inter-provincial power market, the evaluation index system is proposed for the operation effectiveness of inter-provincial electricity market. At last, a case of comparing the short-term and long-term inter-provincial market modes is presented to validate the proposed market evaluation index system.
\end{abstract}

\section{Introduction}

As the deepening of China's electricity market-oriented reform and the confirmation of the second group of spot market pilots, the number of spot market pilot provinces in China is further increasing. Since the market rules in each province are different, the coordination between intra-provincial and inter-provincial markets is more complex, and the operation of inter provincial electricity market is facing more challenges and uncertainties.

The purpose of market evaluation is to make a comprehensive evaluation of market construction and operation. The main objectives of the evaluation include testing whether the market structure is reasonable or not, monitoring whether the market is fully competitive, monitoring the economy and security of market operation, optimizing the spot market rules based on the spot market evaluation results. Through the evaluation of the electricity market, the problems and risks hidden in the market can be clearly observed, and corresponding measures and suggestions are put forward to solve the problems, aiming to improve the fairness and transparency of the market and realize the economic, safe and efficient operation of the electricity market.

Generally, market evaluation outcomes are mainly used in the following scenarios. Firstly, it provides dynamic reference for market operators to timely grasp the market operation status and continuously improve the operation efficiency of market organizations. Secondly, it is to provide basis for relevant government departments to understand the progress and effect of market construction. At last, it is to provide the information of market development trends for the public and the market participants.

In China, the development of electricity market is only at its beginning stage, and the market evaluation system is still under study. In this paper, the international experience of market monitoring and assessment is firstly summarized [1-5]. Then considering the feature of China's inter-provincial power market, the evaluation index system is proposed for the operation effectiveness of inter-provincial electricity market. At last, a case of comparing the near and future inter-provincial market modes is presented to validate the proposed market evaluation index system.

\section{International experience}

\subsection{The U.S.}

The evaluation of the U.S. electricity market are mainly carried out by two organizations. The independent Market Monitoring unit (MMU) evaluates and analyzes the market power, market price, investment trend and competition; the Federal Energy Regulatory Commission (FERC) evaluates the performance of wholesale markets. MMU can be an internal independent supervision department or a third-party independent supervision organization. At present, except for CAISO and SPP, the rest of ISOs in the United States are supervised and evaluated by independent third-party supervision organizations. The PJM evaluation system is based on the SCP paradigm of industrial economics to evaluate the market operation. PJM focuses on the evaluation of market behavior and market manipulation to prevent the formation of market monopoly.

\subsection{Europe}

The electricity markets of European countries are mainly regulated and evaluated by the National Energy

\footnotetext{
${ }^{\text {a } C o r r e s p o n d i n g ~ a u t h o r: ~ z h a o z h e n g @ s g e r i . s g c c . c o m . c n ~}$
} 
Regulatory Agency (NRA). At the EU level, according to the third energy act of the EU, the European energy regulatory agency cooperation committee (ACER) and the Council of European Energy Regulatory agencies (CEER) are established. NRA issues market assessment and regulatory reports, and needs to submit annual reports to ACER every year. ACER forms the annual power market monitoring report based on the reports submitted by NRA and the other useful data from European transmission operators (Entso-e) and power exchange centers. Taking the Nordic electricity market as example, its monitoring report focuses on the generation, energy consumption, transmission, wholesale and retail markets in the Nordic market, as well as the potential development in future.

\subsection{Australia.}

The Australian National Electricity Act requires the Australian Energy Authority (AER) to evaluate the national wholesale electricity market (NEM) at least every two years. The evaluation criteria concerns about whether there is effective competition in NEM, and whether the market has negative effects on the operation of market and the realization of the target of national electricity market. AER uses the SCP model to evaluate NEM comprehensively. The evaluation index system includes market structure, market behavior and market effect.

To sum up, market benefit and efficiency are the key of electricity market evaluation. The goal of building the electricity market is to maximize the social benefits and improve the operation efficiency of the power industry by using market-oriented means. Therefore, the evaluation of market benefit and market efficiency is very important. It is the wind vane to test the effectiveness of market construction. The evaluation index design of electricity market should be adapted to local conditions. Nordic electricity market involves a large number of cross regional transactions, so the cross regional transaction volume and tie-lines are evaluated. Due to the recent increase of market concentration in Australian national electricity market, many indicators are designed to assess the barriers of market entry and withdrawal.

\section{Design of evaluation index system of China's inter-provincial electricity market}

\subsection{Feature of China's inter-provincial electricity market}

The economic and social development of China takes Province as the main body, and the power industry has formed a provincial based power supply pattern for a long time. Energy and power development planning, economic operation and safety production are all managed in accordance with the provincial implementation. At the same time, the unbalanced distribution of energy resources and load and the requirements for the development of renewable energy objectively determine that the unified power market system of China should start with the "two-tier operation" of the inter-provincial and provincial markets, gradually break the barriers of inter provincial market and realize the optimization of energy resources allocation in a wide range.

The inter-provincial market is positioned to ensure the implementation of national energy strategy, realize the optimization of resources allocation in a large range, promote the consumption of renewable energy, and establish resource allocation market. The mid and longterm power energy trading among provinces is positioned to meet the national energy development strategy requirements, realize the wide-range optimization of energy resources allocation and ensure the consumption of renewable energy. The spot market trading among provinces is positioned to realize the mutual benefit of the power surplus and shortage in the cross province through the market mechanism, and to maximize the utilization of inter provincial transmission capacity to promote the large-scale consumption of renewable energy.

\subsection{Inter-provincial electricity market index system}

The evaluation of the effectiveness of inter provincial market mainly includes willingness to participate, transaction scale, price level, clean energy consumption, resource optimization allocation and transmission optimization utilization.

(1) Participation willingness

This indicator reflects whether the market participates in the market is active or not, and whether the proportion of the number of market participants in the market in line with the transaction is reasonable.

(2) Trading scale

This indicator reflects whether the electricity volume of inter provincial transaction, including medium and long term (annual, monthly and monthly), spot trading and proportion are reasonable; whether the proportion of market-oriented transaction electricity in the inter provincial transaction power is reasonable; Whether the proportion of the total transaction electricity in the provincial electricity market to the total electricity volume in the national electricity market is reasonable; Whether the electricity volume of the provincial transactions (including medium and long term and spot, export and import) is reasonable; Whether the electricity consumption of the trading mode (bilateral negotiation, centralized bidding, rolling matching and listing transaction) is reasonable.

(3) Price level

This indicator mainly analyzes the overall price level, price fluctuation, price rationality and price correlation. Whether the price signals (including medium and longterm prices and spot prices) of inter provincial market are distorted, whether the price conduction is smooth and whether the released dividend is reasonable; Whether the 
mid and long-term market between provinces can effectively guide the optimal allocation of power resources among provinces; Whether the spot market between provinces can play a role in regulating the power deviation in the medium and long term.

(4) Clean energy

To measure whether the market mechanism and transaction varieties between provinces promote the consumption of clean energy, and whether the proportion of the consumption of clean energy among provinces to the total consumption of the national power market is reasonable.

(5) Resource allocation

To measure whether it can promote the optimal allocation of power resources nationwide. To quantify the optimal allocation demand of resources among provinces, the indicators such as the power generation cost, the load level of power purchasing and transmission provinces, the declared demand of electricity and the price of power, the weight completion of the responsibility for renewable energy power consumption in power purchase and transmission provinces can be used to determine whether the distribution of economic tide flow is reasonable; Whether the change rate of the annual utilization hours of the cross regional and provincial lines is reasonable.

(6) Transmission utilization

To measure whether optimizing the utilization of inter provincial transmission lines is conducive to improving the utilization rate of inter provincial transmission and reducing congestion.

Table 1. Evaluation indices of market effectiveness

\begin{tabular}{|c|c|}
\hline $\begin{array}{c}\text { 1st Level } \\
\text { Evaluation Index }\end{array}$ & $\begin{array}{c}\text { 2nd Level } \\
\text { Evaluation } \\
\text { Index }\end{array}$ \\
\hline \multirow{2}{*}{ Willingness } & Generator participation \\
\hline & Users participation \\
\hline \multirow{3}{*}{ Trading scale } & Overall \\
\hline & Trading varieties \\
\hline & Trading methods \\
\hline \multirow{4}{*}{ Price level } & Overall \\
\hline & Fluctuation \\
\hline & Rationality \\
\hline & Correlation \\
\hline \multirow{2}{*}{ Clean energy } & Trading varieties \\
\hline & Consumption \\
\hline \multirow{2}{*}{$\begin{array}{l}\text { Resource } \\
\text { allocation }\end{array}$} & Allocation demand \\
\hline & Load flow distribution \\
\hline \multirow{2}{*}{$\begin{array}{l}\text { Transmission } \\
\text { utilization }\end{array}$} & Utilization rate \\
\hline & Congestion \\
\hline
\end{tabular}

\section{Case study}

In this case study, the short-term and long-term modes of inter-provincial electricity markets are modeled and simulated, and the proposed inter-provincial market evaluation indices are used to evaluate the benefit and effectiveness of the two market modes.

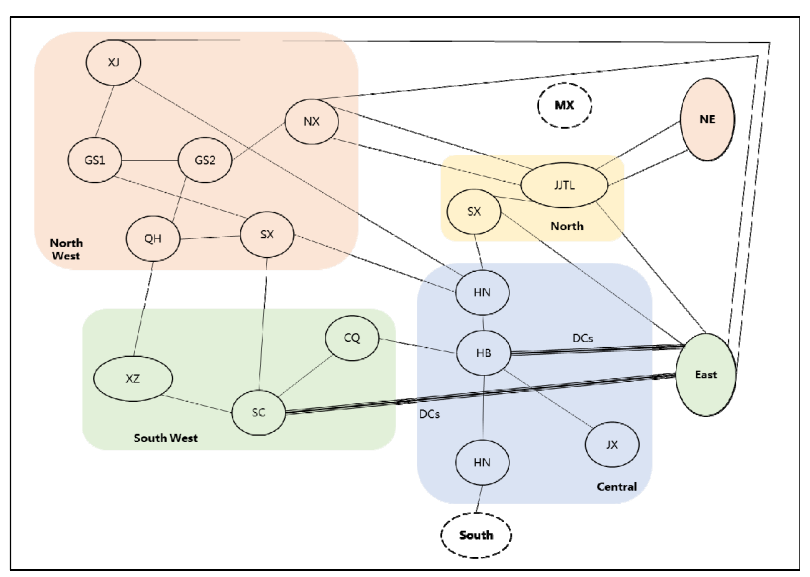

Figure 1. Diagram of inter-provincial market

To model the inter-provincial market, a single province is modeled as a market node, and there are 28 nodes in total. All nodes are interconnected by crossregional and cross-provincial transmission lines. Generators and loads are connected to each node. In dayahead market, generators and load submit their bid and offer data. Then an optimal market clearing to maximize the social welfare is executed considering the power system operation security constraints.

For the short-term mode, the power energy trading is mainly organized on DC transmissions, and only a portion of the provincial generation participates in the inter provincial trading. For the mid-term mode, the inter-provincial trading is implemented on the AC-DC hybrid network, and the whole generation within the province are able to participate in inter-provincial trading.

Table 2. Market effectiveness comparison of short-term and long-term market modes

\begin{tabular}{|c|c|c|}
\hline Trading volume & Short-term mode & Long-term mode \\
\hline Price level & $\begin{array}{c}\text { Average price } \\
291.8 \\
\text { Yuan/MWh }\end{array}$ & $\begin{array}{c}\text { Average price } \\
269.6 \\
\text { Yuan/MWh }\end{array}$ \\
\hline $\begin{array}{c}\text { Clean energy } \\
\text { consumption }\end{array}$ & $701 \mathrm{GWh}$ & $926 \mathrm{GWh}$ \\
\hline $\begin{array}{c}\text { Resource } \\
\text { allocation }\end{array}$ & $\begin{array}{c}\text { Social welfare } \\
0.25 \text { billion Yuan }\end{array}$ & $\begin{array}{c}\text { Social welfare } 0.39 \\
\text { billion Yuan }\end{array}$ \\
\hline $\begin{array}{c}\text { Transmission } \\
\text { utilization }\end{array}$ & $\begin{array}{c}\text { DC transmission } \\
\text { has } 1618 \mathrm{GWh} \\
\text { traded }\end{array}$ & $\begin{array}{c}\text { AChid has } 2321 \\
\text { GWh traded, } \\
\text { where DC } \\
\text { accounts for } 32 \% \\
\text { and has more } \\
\text { space in future }\end{array}$ \\
\hline
\end{tabular}

Through the comparative analysis, it can be seen that the overall comprehensive benefit of the proposed longterm market model is better than that of the short-term market model. Therefore, it is suggested that with the 
development of transmission and distribution price, cross subsidy, power grid structure, power and electricity balance pattern, power supply and demand balance pattern and so on, it should promote the gradual integration of inter provincial and intra provincial markets, expand the scope of market participants, enrich trading varieties, and gradually step into long-term market mode.

\section{Case study}

Based on the international market evaluation experience and the situation of China's inter-provincial market, this paper studies and designs the evaluation system framework of China's inter-provincial market. The evaluation system is proposed to comprehensively reflect the market environment, market construction progress and market operation, and adopt the combination of quantitative and qualitative methods to form the overall evaluation of the inter-provincial market, so as to provide reference for further improving the rules and mechanisms of the market.

\section{Acknowledgements}

This work is supposed in part by the Science and Technology Project of SGCC-Research on "Key Technologies of the Operation Monitoring and Analysis of Spot Electricity Market under China's New Reform" (Grant 5108-201957038A-0-0-00)

\section{References}

1. Chen Qing, Yang Junwei, Huang Yuanming, Lu en, Wang Yi. Overview of market power monitoring and mitigation mechanism in foreign power markets [J]. China Southern Power Grid technology, 2018,12 (12): 9-15.

2. Li Youliang, $\mathrm{Wu} \mathrm{Xu,} \mathrm{Liang} \mathrm{Xiao,} \mathrm{Zhang} \mathrm{Wei,} \mathrm{Xie}$ Xiaoyan. Discussion on the construction scheme of provincial electric power spot market [J]. Electrical engineering, 2020 (09): 61-65 .

3. Wang Yong, you Daning, Fang Guanghua, Zhang Guoqiang, Wang Jin, Kuang Honghui. Mechanism design and trial operation analysis of Shandong electric power spot market [J]. China electric power, 2020,53 (09): 38-46.

4. Du Limin, Shi Jinchuan. Market power monitoring in power market: a review [J]. Journal of Zhejiang University (HUMANITIES AND SOCIAL SCIENCES), 2007 (04): 153-162.

5. Wang Peng. Research on regional power market monitoring and power audit [J]. China Science and technology investment, 2013 (26): 104 [11] Fu Kaibao, Zeng Xiang. Analysis of the status of electricity market reform and policy recommendations[J]. Macroeconomic Management, 2018(1): 49-54. 